\title{
MEAN-VALUES AND HARMONIC POLYNOMIALS
}

\author{
BY \\ E. F. BECKENBACH AND MAXWELL READE \\ INTRODUCTION
}

0.1. It is well known that if the function $f(x, y)$ is harmonic in a finite domain (non-null connected open set) $D$, then at each point $\left(x_{0}, y_{0}\right)$ in $D$, $f(x, y)$ satisfies the equation

$$
f\left(x_{0}, y_{0}\right)=\frac{1}{\pi r^{2}} \iint_{D\left(x_{0}, y_{0} ; r\right)} f\left(x_{0}+\xi, y_{0}+\eta\right) d \xi d \eta
$$

for each circular disc

$$
D\left(x_{0}, y_{0} ; r\right): \xi^{2}+\eta^{2}=\left(x-x_{0}\right)^{2}+\left(y-y_{0}\right)^{2} \leqq r^{2}
$$

lying in $D$. Conversely, if $f(x, y)$ is superficially summable in the interior of a finite domain $D$, and if (1) holds for each point $\left(x_{0}, y_{0}\right)$ and each disc $D\left(x_{0}, y_{0} ; r\right)$ about $\left(x_{0}, y_{0}\right)$ in $D$, then $f(x, y)$ is harmonic in $D\left({ }^{1}\right)$.

It follows that (1) may be taken as the defining equation for harmonic functions.

0.2. Similarly, if $f(x, y)$ is superficially summable in the interior of a finite simply-connected domain $D$, and if $f(x, y)$ is summable on each circle

$$
C\left(x_{0}, y_{0} ; r\right): \xi^{2}+\eta^{2}=\left(x-x_{0}\right)^{2}+\left(y-y_{0}\right)^{2}=r^{2}
$$

lying in $D$, then a necessary and sufficient condition that $f(x, y)$ be harmonic in $D$ is that at each point $\left(x_{0}, y_{0}\right)$ in $D, f(x, y)$ satisfy the equation

$$
f\left(x_{0}, y_{0}\right)=\frac{1}{2 \pi r} \int_{C\left(x_{0}, y_{0} ; r\right)} f\left(x_{0}+\xi, y_{0}+\eta\right) d s
$$

for each circle $C\left(x_{0}, y_{0} ; r\right)$ in $D$.

As with (1), (2) may be taken as the defining equation for harmonic functions.

0.3. The following theorem is analogous to a result of Beckenbach and Rado concerning subharmonic functions $\left({ }^{2}\right)$.

THEOREM 1. If $f(x, y)$ is continuous in a finite domain $D$, then a necessary

Presented to the Society, December 30, 1941; received by the editors March 20, 1942.

(1) See E. Levi, Supra una proprieta caratteristica delle funzione armoniche, Atti della Reale Academia Lincei vol. 18 (1909) pp. 10-15, and L. Tonelli, Sopra una proprietd caratteristica delle funzione armoniche, ibid. pp. 577-582.

(2) E. F. Beckenbach and Tibor Rado, Subharmonic functions and surfaces of negative curvature, Trans. Amer. Math. Soc. vol. 35 (1933) pp. 662-674. 
and sufficient condition that $f(x, y)$ be harmonic in $D$ is that for each point $\left(x_{0}, y_{0}\right)$ in $D$, the equation

(3) $\frac{1}{2 \pi r} \int_{C\left(x_{0}, y_{0} ; r\right)} f\left(x_{0}+\xi, y_{0}+\eta\right) d s=\frac{1}{\pi r^{2}} \iint_{D\left(x_{0}, y_{0} ; r\right)} f\left(x_{0}+\xi, y_{0}+\eta\right) d \xi d \eta$ hold for all $D\left(x_{0}, y_{0} ; r\right)$ in $D$.

Proof. If $f(x, y)$ is harmonic in $D$, then (3) follows from (1) and (2).

To prove that (3) is a sufficient condition that $f(x, y)$ be harmonic in $D$, we consider the circular average $\left({ }^{8}\right)$

$$
f(x, y ; \rho) \equiv \frac{1}{\pi \rho^{2}} \iint_{D(x, y ; \rho)} f(x+\xi, y+\eta) d \xi d \eta,
$$

which is defined in an open subset of $D$. For $D\left(x_{0}, y_{0} ; \rho\right)$ in $D$, a computation yields

$$
\begin{aligned}
\frac{d}{d \rho} f\left(x_{0}, y_{0} ; \rho\right)=\frac{2}{\rho}\left[\frac{1}{2 \pi \rho} \int_{C\left(x_{0}, y_{0} ; \rho\right)} f\left(x_{0}+\xi, y_{0}+\eta\right) d s\right. & \\
& \left.-\frac{1}{\pi \rho^{2}} \iint_{D\left(x_{0}, y_{0} ; \rho\right)} f\left(x_{0}+\xi, y_{0}+\eta\right) d \xi d \eta\right],
\end{aligned}
$$

which, with (3), shows that $f\left(x_{0}, y_{0} ; \rho\right)$ is independent of $\rho$. But since $f(x, y)$ is continuous, we have $f(x, y ; \rho) \rightrightarrows f(x, y)$, as $\rho \rightarrow 0$, on each closed subset of $D$, so that

$$
f\left(x_{0}, y_{0}\right)=f\left(x_{0}, y_{0} ; r\right)=\frac{1}{\pi r^{2}} \iint_{D\left(x_{0}, y_{0} ; r\right)} f\left(x_{0}+\xi, y_{0}+\eta\right) d \xi d \eta
$$

for each $D\left(x_{0}, y_{0} ; r\right)$ in $D$. Therefore $f(x, y)$ is harmonic in $D$.

If we should assume only that $f(x, y)$ is superficially summable and satisfies (3), then it would not follow that $f(x, y)$ is harmonic; consider, for example, the function which vanishes identically except at the origin, where it assumes the value 1 .

0.4. The right-hand members in (1) and (2) are areal and peripheral averages (mean-values), respectively; in each instance the range of integration is circular. The question arises as to the nature of the functions which are defined by relations similar to (1), (2) and (3), in which the geometric figure is square, elliptic, and so on (4).

In this paper we delineate the classes of functions defined by the condition

() See $\S 1.3$ below.

(4) Cf. W. Brödel, Funktionen mit Gaussischer Mittelwerteigenschaft für konvexe Kurven und Bereiche, Deutsche Mathematik, vol. 4 (1939) pp. 3-15. By combining our methods with his, one can simplify the proofs of some of his results concerning general mean-values. 
that their averages over regular polygons of $n$ sides satisfy conditions similar to (1), (2) and (3)(5).

0.5. Since a circle may be considered as a limit of a sequence of circumscribed (or inscribed) regular polygons, the results of this introductory section may be considered as limiting cases of some of the results obtained below.

\section{LEMMAS}

1.1. We recall that if $n \geqq 2$, then for all angles $\psi$ we have

$$
\begin{aligned}
\sum_{m=0}^{n-1} \sin \left(\psi+\frac{2 \pi m}{n}\right)=\sum_{m=0}^{n-1} \cos \left(\psi+\frac{2 \pi m}{n}\right) & =0, \\
\sum_{m=0}^{n-1} \sin \left(\psi+\frac{2 \pi m}{n}\right) \cos \left(\psi+\frac{2 \pi m}{n}\right) & =0, \\
\sum_{m=0}^{n-1} \sin ^{2}\left(\psi+\frac{2 \pi m}{n}\right)=\sum_{m=0}^{n-1} \cos ^{2}\left(\psi+\frac{2 \pi m}{n}\right) & =n / 2 .
\end{aligned}
$$

Thus

$$
\begin{aligned}
\sum_{m=0}^{n-1}\left[\cos \left(\psi+\frac{2 \pi m}{n}\right)+i \sin (\psi\right. & \left.\left.+\frac{2 \pi m}{n}\right)\right] \\
& =(\cos \psi+i \sin \psi) \sum_{m=0}^{n-1}\left(\cos \frac{2 \pi m}{n}+i \sin \frac{2 \pi m}{n}\right)
\end{aligned}
$$

and this vanishes since the sum of the $n$th roots of unity is zero. The remaining formulas in (4), (5) and (6) can be established in a similar way.

More generally, for $n \geqq 1$,

$$
\sum_{m=0}^{n-1}\left[\cos \left(\psi+\frac{2 \pi k m}{n}\right)+i \sin \left(\psi+\frac{2 \pi k m}{n}\right)\right]=n \delta_{k, n}(\cos \psi+i \sin \psi)
$$

where $\delta_{k, n}=1$ if $k$ is an integral multiple of $n$, and where $\delta_{k, n}=0$ otherwise $\left(^{6}\right)$.

1.2. $P_{n}\left(x_{0}, y_{0} ; r ; \phi\right), n \geqq 3$, shall denote the closed finite region bounded by the regular $n$-gon $p_{n}\left(x_{0}, y_{0} ; r ; \phi\right)$, whose center is at $\left(x_{0}, y_{0}\right)$ and whose inscribed circle has radius $r ; \phi$ denotes the angle from $R$ to $N,-\pi / n \leqq \phi<\pi / n$, where $R$ is the ray extending horizontally to the right from $\left(x_{0}, y_{0}\right)$ and $N$ is the exterior normal at the point where $R$ emerges from the polygon. $\left|P_{n}\left(x_{0}, y_{0} ; r ; \phi\right)\right|$ shall denote the area of $P_{n}\left(x_{0}, y_{0} ; r ; \phi\right)$ and $\left|p_{n}\left(x_{0}, y_{0} ; r ; \phi\right)\right|$ shall denote the length of $p_{n}\left(x_{0}, y_{0} ; r ; \phi\right)$.

We enumerate the sides of $p_{n}\left(x_{0}, y_{0} ; r ; \phi\right)$ in counter-clockwise fashion, $s_{0}, s_{1}, \cdots, s_{n-1}$, where $s_{0}$ is the side to which $N$ is normal.

$\left(^{5}\right) \mathrm{J}$. L. Walsh considered finite averages over the vertices of regular $n$-gons, obtaining results similar to some of the results obtained in this paper, in his article $A$ mean-value theorem for polynomials and harmonic polynomials, Bull. Amer. Math. Soc. vol. 42 (1936) pp. 923-930.

$\left({ }^{6}\right)$ Walsh, loc. cit. p. 924. 
1.3. LEMma 1. If $f(x, y)$ is superficially summable in the interior of a finite domain $D$, if $n$ is a fixed integer, $n \geqq 3$, and if for each point $\left(x_{0}, y_{0}\right)$ in $D$ the equation

$$
f\left(x_{0}, y_{0}\right)=\frac{1}{\left|P_{n}\left(x_{0}, y_{0} ; r ; 0\right)\right|} \iint_{P_{n}\left(x_{0}, y_{0} ; r ; 0\right)} f\left(x_{0}+\xi, y_{0}+\eta\right) d \xi d \eta
$$

holds for each $P_{n}\left(x_{0}, y_{0} ; r ; 0\right)$ in $D$, then $f(x, y)$ is harmonic in $D\left({ }^{7}\right)$.

Proof. For any function $F(x, y)$, defined and superficially summable in the interior of $D$, the areal averaging function

$$
F(x, y ; r) \equiv \frac{1}{\left|P_{n}(x, y ; r ; 0)\right|} \iint_{P_{n}(x, y ; r ; 0)} F(x+\xi, y+\eta) d \xi d \eta
$$

is one degree smoother than $F(x, y)$ in the open subset $D_{r}$ of $D$, where $F(x, y ; r)$ is defined; that is, if $F(x, y)$ is superficially summable in the interior of $D$, then (for $r$ fixed) $F(x, y ; r)$ is (at least) continuous in $D_{r}$, or if $F(x, y)$ has continuous partial derivatives of the $n$th order in $D$, then $F(x, y ; r)$ has continuous partial derivatives of the $(n+1)$ st order in $D_{r}\left({ }^{8}\right)$. Hence it follows by a simple induction that if the summable function $f(x, y)$ satisfies (8) at each point $\left(x_{0}, y_{0}\right)$ in $D$, for each $P_{n}\left(x_{0}, y_{0} ; r ; 0\right)$ lying in $D$, then $f(x, y)$ has continuous partial derivatives of all orders in $D$.

Accordingly, we may use the finite Taylor expansion

$$
f\left(x_{0}+\xi, y_{0}+\eta\right)=f\left(x_{0}, y_{0}\right)+\sum_{k=1}^{2} \frac{1}{k !}\left[\left(\xi \frac{\partial}{\partial x}+\eta \frac{\partial}{\partial y}\right)^{k} f\right]+o\left(\zeta^{2}\right),
$$

for $f(x, y)$, about the point $\left(x_{0}, y_{0}\right)$; here

$$
\zeta^{2}=\xi^{2}+\eta^{2}
$$

$$
\xi \frac{\partial}{\partial x}+\eta \frac{\partial}{\partial y}
$$

is a differential operator, the partial derivatives are evaluated at $\left(x_{0}, y_{0}\right)$, and $o\left(r^{\alpha}\right)$ denotes a function (not always the same function) such that

$$
\lim _{r \rightarrow 0} \frac{o\left(r^{\alpha}\right)}{r^{\alpha}}=0
$$

The side $s_{m}$ of $P_{n}\left(x_{0}, y_{0} ; r ; 0\right)$ can be represented by the polar equation

$$
s_{m}: \quad \rho=r \sec (\theta-2 \pi m / n), \quad(2 m-1) \pi / n \leqq \theta \leqq(2 m+1) \pi / n ;
$$

(7) In the proof we use only the weaker assumption that the difference of the two members in (8) is (uniformly) $o\left(r^{4}\right)$ in $D$.

(8) For a list of the principal properties of averaging functions, see H. E. Bray, Proof of a formula for an area, Bull. Amer. Math. Soc. vol. 29 (1923) pp. 264-270. 
that is

$$
s_{m}: \quad \rho=r \sec \psi, \quad \psi=\theta-2 \pi m / n, \quad-\pi / n \leqq \psi \leqq \pi / n .
$$

Hence we have

$$
\begin{array}{r}
\iint_{P_{n}\left(x_{0}, y y_{0} ; ; 0\right)} f\left(x_{0}+\xi, y_{0}+\eta\right) d \xi d \eta=\left|P_{n}\left(x_{0}, y_{0} ; r ; 0\right)\right| f\left(x_{0}, y_{0}\right) \\
+\sum_{m=0}^{n-1} \int_{-\pi / n}^{\pi / n} \int_{0}^{r \sec \psi} \sum_{k=1}^{2} \frac{1}{k !}\left\{\left[\rho \cos \left(\psi+\frac{2 \pi m}{n}\right) \frac{\partial}{\partial x}\right.\right. \\
\left.\left.+\rho \sin \left(\psi+\frac{2 \pi m}{n}\right) \frac{\partial}{\partial y}\right]^{k} f\left(x_{0}, y_{0}\right)\right\} \rho d \rho d \psi+o\left(r^{4}\right) .
\end{array}
$$

Applying (4), (5) and (6), we obtain

$$
\begin{aligned}
\iint_{P_{n}\left(x_{0}, y_{0} ; r ; 0\right)} f\left(x_{0}+\xi,\right. & \left.y_{0}+\eta\right) d \xi d \eta=\left|P_{n}\left(x_{0}, y_{0} ; r ; 0\right)\right| f\left(x_{0}, y_{0}\right) \\
& +\frac{n r^{4}}{8}\left(\frac{1}{3} \tan ^{3} \frac{\pi}{n}+\tan \frac{\pi}{n}\right) \Delta f\left(x_{0}, y_{0}\right)+o\left(r^{4}\right),
\end{aligned}
$$

where

$$
\Delta \equiv \frac{\partial^{2}}{\partial x^{2}}+\frac{\partial^{2}}{\partial y^{2}}
$$

is the Laplacian operator. The lemma now follows from (8) and (10), which yield the equation $\Delta f(x, y)=0$.

1.4. If $f(x, y)$ is harmonic in a finite domain $D$, then $f(x, y)$ may be expanded in a Fourier series about each point $\left(x_{0}, y_{0}\right)$ in $D$ :

$$
\begin{aligned}
f(x, y) & =f\left(x_{0}+\rho \cos \theta, y_{0}+\rho \sin \theta\right) \\
& =f\left(x_{0}, y_{0}\right)+\sum_{k=1}^{\infty} \rho^{k}\left(a_{k} \cos k \theta+b_{k} \sin k \theta\right) .
\end{aligned}
$$

LEMMa 2. If $f(x, y)$ is harmonic in a finite domain $D$, then for each $P_{n}\left(x_{0}, y_{0}\right.$; $r ; 0)$ such that $D\left(x_{0}, y_{0} ; r \sec \pi / n\right)$ is in $D$ we have

$$
\begin{aligned}
\iint_{P_{n}\left(x_{0}, y_{0} ; r ; 0\right)} f\left(x_{0}+\xi, y_{0}+\eta\right) d \xi d \eta & =\left|P_{n}\left(x_{0}, y_{0} ; r ; 0\right)\right| f\left(x_{0}, y_{0}\right) \\
+ & n \sum_{k=1}^{\infty} \frac{a_{k n} r^{k n+2}}{k n+2} \int_{-\pi / n}^{\pi / n} \sec ^{k n+2} \psi \cos k n \psi d \psi .
\end{aligned}
$$

Proof. Using (7), (9) and (11), we obtain 


$$
\begin{aligned}
\iint_{P_{n}\left(x_{0}, y_{0} ; r ; 0\right)} f\left(x_{0}\right. & \left.+\xi, y_{0}+\eta\right) d \xi d \eta=\left|P_{n}\left(x_{0}, y_{0} ; r ; 0\right)\right| f\left(x_{0}, y_{0}\right) \\
& +n \int_{-\pi / n}^{\pi / n} \int_{0}^{r \sec \psi} \sum_{k=1}^{\infty} \rho^{k n}\left(a_{k n} \cos k n \psi+b_{k n} \sin k n \psi\right) \rho d \rho d \psi .
\end{aligned}
$$

Now (12) follows from the fact that $\sin \theta$ is an odd function, while $\cos \theta$ and $\sec \theta$ are even functions, of $\theta$.

\section{Areal mean-values}

2.1. The real and imaginary parts of $(x+i y)^{n}$ are basic homogeneous harmonic polynomials of degree $n$ in the variables $x, y$. We shall denote these polynomials by $H_{1, n}(x, y)$ and $H_{2, n}(x, y)$, respectively. Any homogeneous harmonic polynomial of degree $n$ in $x, y$ is of the form

$$
a H_{1, n}(x, y)+b H_{2, n}(x, y)
$$

where $a$ and $b$ are constants.

2.2. THEOREM 2. If $f(x, y)$ is superficially summable in the interior of a finite domain $D$, and if $n$ is a fixed integer, $n \geqq 3$, then a necessary and sufficient condition that for each point $\left(x_{0}, y_{0}\right)$ in $D$, the equation

$$
f\left(x_{0}, y_{0}\right)=\frac{1}{\left|P_{n}\left(x_{0}, y_{0} ; r ; 0\right)\right|} \iint_{P_{n}\left(x_{0}, y_{0} ; r ; 0\right)} f\left(x_{0}+\xi, y_{0}+\eta\right) d \xi d \eta
$$

hold for each $P_{n}\left(x_{0}, y_{0} ; r ; 0\right)$ in $D$ is that $f(x, y)$ be a harmonic polynomial of degree at most $n$, of the form

$$
f(x, y)=B_{0}+\sum_{k=1}^{n-1}\left[A_{k} H_{1, k}(x, y)+B_{k} H_{2, k}(x, y)\right]+B_{n} H_{2, n}(x, y),
$$

where $A_{k}$ and $B_{k}$ are constants, $k=0,1, \cdots, n$.

Necessity. If (13) holds, then, by Lemma $1, f(x, y)$ is harmonic; consequently, by (13) and Lemma 2, we have

$$
\sum_{k=1}^{\infty} \frac{a_{k n} r^{k n+2}}{k n+2} \int_{-\pi / n}^{\pi / n} \sec ^{k n+2} \psi \cos k n \psi d \psi=0 .
$$

Since (15) holds for all sufficiently small $r$, the coefficient of each power of $r$ must vanish; in particular we have

$$
\frac{a_{n}}{n+2} \int_{-\pi / n}^{\pi / n} \sec ^{n+2} \psi \cos n \psi d \psi=0
$$

Now

$$
\int_{-\pi / n}^{\pi / n} \sec ^{n+2} \psi \cos n \psi d \psi<0
$$


as one readily sees by an inspection of the graphs of the functions $\sec \psi$ and $\cos n \psi$. Therefore (16) yields

$$
a_{n}=\frac{1}{n !} \frac{\partial^{n}}{\partial x^{n}} f\left(x_{0}, y_{0}\right)=0 .
$$

But (13) holds at each point $\left(x_{0}, y_{0}\right)$ in $D$, so that

$$
\frac{\partial^{n} f(x, y)}{\partial x^{n}}=0
$$

holds throughout $D$. By a simple induction we obtain, from (17) and the Laplace equation $\Delta f(x, y)=0$,

(18) $\frac{\partial^{n+k} f(x, y)}{\partial x^{s} \partial y^{t}}=0, s+t=n+k ; s, t=0,1, \cdots, n+k ; k=1,2, \cdots$.

Now (17) and (18) imply (14).

Sufficiency. If $f(x, y)$ is of the form $(14)$, then $f(x, y)$ can be continued harmonically so as to be defined and harmonic in the entire $x, y$-plane. Now (13) follows from (12), (17) and (18).

2.3. TheOREM 3. If $f(x, y)$ is superficially summable in the interior of a finite domain $D$, if $n$ is a fixed integer, $n \geqq 3$, and if $\phi_{0}$ is fixed, $-\pi / n \leqq \phi_{0}<\pi / n$, then a necessary and sufficient condition that for each point $\left(x_{0}, y_{0}\right)$ in $D$, the equation

$$
f\left(x_{0}, y_{0}\right)=\frac{1}{\left|P_{n}\left(x_{0}, y_{0} ; r ; \phi_{0}\right)\right|} \iint_{P_{n}\left(x_{0}, y_{0} ; r ; \phi_{0}\right)} f\left(x_{0}+\xi, y_{0}+\eta\right) d \xi d \eta
$$

hold for each $P_{n}\left(x_{0}, y_{0} ; r ; \phi_{0}\right)$ in $D$ is that $f(x, y)$ be a harmonic polynomial of degree at most $n$, of the form

$$
\begin{aligned}
f(x, y)=B_{0} & +\sum_{k=1}^{n-1}\left[A_{k} H_{1, k}(x, y)+B_{k} H_{2, k}(x, y)\right] \\
& +B_{n}\left[H_{1, n}(x, y) \sin n \phi_{0}+H_{2, n}(x, y) \cos n \phi_{0}\right],
\end{aligned}
$$

where $A_{k}$ and $B_{k}$ are constants, $k=0,1, \cdots, n$.

Proof. If we make the transformation of coordinates $x^{\prime}+i y^{\prime}=(x+i y) e^{i \phi_{0}}$, then Theorem 3 follows at once from Theorem 2.

2.4. THEOREM 4. If $f(x, y)$ is superficially summable in the interior of a finite domain $D$, and if $n$ is a fixed integer, $n \geqq 3$, then a necessary and sufficient condition that for each point $\left(x_{0}, y_{0}\right)$ in $D$, the equation

$$
f\left(x_{0}, y_{0}\right)=\frac{1}{\left|P_{n}\left(x_{0}, y_{0} ; r ; \phi\right)\right|} \iint_{P_{n}\left(x_{0}, y_{0} ; r ; \phi\right)} f\left(x_{0}+\xi, y_{0}+\eta\right) d \xi d \eta
$$


hold for each $P_{n}\left(x_{0}, y_{0} ; r ; \phi\right)$ in $D$, is that $f(x, y)$ be a harmonic polynomial of degree at most $n-1\left({ }^{9}\right)$.

Proof. The theorem follows from Theorem 3 and the fact that while the class of harmonic polynomials of the form (20) is not invariant under rotations of the plane, the class of harmonic polynomials of degree less than $n$ is invariant under these rotations.

\section{Peripheral mean-values}

3.1. TheOREM 5. If $f(x, y)$ is summable on each $P_{n}(x, y ; r ; 0)$ and on each $p_{n}(x, y ; r ; 0)$ lying in a finite simply-connected domain $D$, where $n$ is a fixed integer, $n \geqq 3$, then a necessary and sufficient condition that for each point $\left(x_{0}, y_{0}\right)$ in $D$ the equation

$$
f\left(x_{0}, y_{0}\right)=\frac{1}{\left|p_{n}\left(x_{0}, y_{0} ; r ; 0\right)\right|} \int_{p_{n}\left(x_{0}, y_{0} ; r ; 0\right)} f\left(x_{0}+\xi, y_{0}+\eta\right) d s
$$

hold for each $p_{n}\left(x_{0} y_{0} ; r ; 0\right)$ in $D$ is that $f(x, y)$ be a harmonic polynomial of the form $(14)\left({ }^{10}\right)$.

NeCEssity. If we multiply both members of $(21)$ by $\left|p_{n}\left(x_{0}, y_{0} ; r ; 0\right)\right|$ and integrate with respect to $r$, and then apply the theorem of Fubini to the superficially summable function $f(x, y)$, we obtain (13) for each point $\left(x_{0}, y_{0}\right)$ in $D$, for each $P_{n}\left(x_{0}, y_{0} ; r ; 0\right)$ lying in $D$. Hence, by Theorem $2, f(x, y)$ is of the form (14).

Sufficiency. If $f(x, y)$ is given by (14), then, by Theorem 2, (13) holds. Differentiating both sides of (13) with respect to $r$, we obtain

$$
\begin{aligned}
\frac{1}{\left|p_{n}\left(x_{0}, y_{0} ; r ; 0\right)\right|} \int_{p_{n}\left(x_{0}, y_{0} ; r ; 0\right)} f\left(x_{0}+\xi, y_{0}+\eta\right) d s \\
=\frac{1}{\left|P_{n}\left(x_{0}, y_{0} ; r ; 0\right)\right|} \iint_{P_{n}\left(x_{0}, y_{0} ; r ; 0\right)} f\left(x_{0}+\xi, y_{0}+\eta\right) d \xi d \eta,
\end{aligned}
$$

which, with (13), implies (21).

3.2. The following two theorems are analogous to Theorems 3 and 4 , respectively.

THEOREM 6. If $f(x, y)$ is summable on each $P_{n}\left(x, y ; r ; \phi_{0}\right)$ and on each $p_{n}\left(x_{0}, y_{0} ; r ; \phi_{0}\right)$ lying in a finite simply-connected domain $D$, where $n$ is a fixed integer, $n \geqq 3$, and where $\phi_{0}$ is fixed, $-\pi / n \leqq \phi_{0}<\pi / n$, then a necessary and sufficient condition that for each point $\left(x_{0}, y_{0}\right)$ in $D$, the equation

$$
f\left(x_{0}, y_{0}\right)=\frac{1}{\left|p_{n}\left(x_{0}, y_{0} ; r ; \phi_{0}\right)\right|} \int_{p_{n}\left(x_{0}, y_{0} ; r ; \phi_{0}\right)} f\left(x_{0}+\xi, y_{0}+\eta\right) d s
$$

( ${ }^{9}$ Cf. Walsh, loc. cit., p. 923, Theorem 3.

(10) Theorems 5-7 actually hold for $n=2$. We have stated them for $n \geqq 3$ to conform with the analogous Theorems $2-4$, respectively. 
hold for each $p_{n}\left(x_{0}, y_{0}, r ; \phi_{0}\right)$ in $D$ is that $f(x, y)$ be a harmonic polynomial of the form (20).

Theorem 7. If $f(x, y)$ is summable on each $P_{n}(x, y ; r ; \phi)$ and on each $p_{n}(x, y ; r ; \phi)$ lying in a finite simply-connected domain $D$, where $n$ is a fixed integer, $n \geqq 3$, then a necessary and sufficient condition that for each point $\left(x_{0}, y_{0}\right)$ in $D$, the equation

$$
f\left(x_{0}, y_{0}\right)=\frac{1}{\left|p_{n}\left(x_{0}, y_{0} ; r ; \phi\right)\right|} \int_{p_{n}\left(x_{0}, y_{0} ; r ; \phi\right)} f\left(x_{0}+\xi, y_{0}+\eta\right) d s
$$

hold for each $p_{n}(x, y ; r ; \phi)$ in $D$ is that $f(x, y)$ be a harmonic polynomial of degree at most $n-1$.

Theorems 6 and 7 follow from Theorems 3 and 4, respectively, in the same way that Theorem 5 follows from Theorem 2 .

3.3. Theorems 5, 6 and 7 are analogous to Theorems 2, 3 and 4, respectively. Similarly, we might give three theorems of the type of Theorem 1 which are analogous to Theorems 2, 3 and 4 . We give the explicit statement of only the last of these:

THEOREM 8. If $f(x, y)$ is continuous in a finite simply-connected domain $D$, then a necessary and sufficient condition that for each point $\left(x_{0}, y_{0}\right)$ in $D$, the equation

$$
\begin{aligned}
& \frac{1}{\left|p_{n}\left(x_{0}, y_{0} ; r ; \phi\right)\right|} \int_{p_{n}\left(x_{0}, y_{0} ; r ; \phi\right)} f\left(x_{0}+\xi, y_{0}+\eta\right) d s \\
& \quad=\frac{1}{\left|P_{n}\left(x_{0}, y_{0} ; r ; \phi\right)\right|} \iint_{P_{n}\left(x_{0}, y_{0} ; r ; \phi\right)} f\left(x_{0}+\xi, y_{0}+\eta\right) d \xi d \eta
\end{aligned}
$$

hold for each $P_{n}\left(x_{0}, y_{0} ; r ; \phi\right)$ lying in $D$, is that $f(x, y)$ be a harmonic polynomial of degree at most $n-1$.

The University of Michigan, ANN ARbor, Mich.

The Ohio State University, Columbus, Оніо 